\title{
sciendo
}

10.2478/AMB-2019-0008

\section{YOGURT DRINK EFFECTIVELY REHYDRATES ATHLETES AFTER A STRENUOUS EXERCISE SESSION}

\author{
M. Niksefat ${ }^{1}$, M. Akbari-Fakhrabadi ${ }^{1}$, Z. Mousavi ${ }^{1}$, V. Ziaee ${ }^{2}$, J. Fallah ${ }^{1}$, A. H. Memari ${ }^{3}$ \\ ${ }^{1}$ Sports Medicine Research Center, Neuroscience Institute, Tehran University of Medical Sciences - Tehran, Iran \\ ${ }^{2}$ Growth and Development Research Center, Pediatrics Department, Tehran University of Medical Sciences \\ - Tehran, Iran \\ ${ }^{3}$ Neuroscience Institute, Tehran University of Medical Sciences - Tehran, Iran
}

\begin{abstract}
Dehydration and electrolyte imbalance as a result of prolonged strenuous exercise leads to poor thermoregulation and impaired muscle performance. Thus, appropriate rehydration during and after exercise with a solution that has a balanced combination of nutrients including electrolytes, carbohydrates and proteins is crucial in preventing the side effects of dehydration. Yogurt drink as a traditional drink with an appropriate nutritious content could be used as an alternative to expensive commercially available drinks for rehydrating athletes after long exercises in warm and humid environments and in developing countries. In this cross-over trial we examined the rehydration potential of yogurt drink in comparison to water and a commercial drink after a strenuous exercise (cycling) session in a hot and humid environment. Blood measurements included blood osmolality, serum glucose, hematocrit and serum electrolytes. Urine measurements included urine volume, osmolality and electrolyte concentrations. The results showed that early after rehydration Yogurt drink compared to water significantly increased urine sodium ( $\mathrm{mmol} / \mathrm{L})(138.8 \pm 93.4 \mathrm{vs} .90 .0 \pm 50.7, P<0.01)$, urine potassium $(\mathrm{mmol} / \mathrm{L})(105.6 \pm 55.1 \mathrm{vs} .35 .8 \pm 22.0, P<0.05)$ and urine chloride $(\mathrm{mmol} / \mathrm{L})$ (113.2 \pm 28.4 vs. $35.8 \pm 25.1 P<0.01)$. This degree of improvement was the same as with the commercial drink for urine sodium, potassium, and chloride. Yogurt drink prevented dehydration-induced changes in the blood as it stabilized the hematocrit and serum sodium, potassium and osmolality within the normal range after exercise. Finally, our results indicated that yogurt drink, as a natural and affordable rehydration option, can be considered to restore fluid and electrolyte losses after strenuous exercises in hot and humid environments.
\end{abstract}

Key words: yogurt drink, dehydration, electrolytes, sports drink, exercise

Corresponding author: Maryam Akbari-Fakhrabadi, Sports Medicine Research Center, Neuroscience Institute, Tehran University of Medical Sciences, Tehran, Iran, e-mail: akbari.maryam88@gmail.com

\section{INTRODUCTION}

trenuous exercise leads to increased heat production due to muscular contraction which in return increases skin blood perfusion and perspiration occurs to regulate the body's temperature [1]. Sweating can lead to loss of fluid and electrolytes, especially sodium which could cause plasma volume contraction [2]. If not compensated, it will disrupt thermoregulatory processes which may end in a vicious cycle. In addition, with no replenishment of fluid and electrolytes, the athlete will encounter increasing fatigue, metabolic changes and cardiovascular strain $[3,4]$. Thus, rehydrating during exercise with a so- 
lution that has a balanced combination of nutrients including electrolytes, carbohydrates and proteins is crucial in preventing the side effects of dehydration. There has been much research on the content of rehydrating drinks for athletes. Besides sodium, replenishment of potassium and magnesium is also crucial in maintaining the physiological hemodynamic parameters, electrolyte balance and muscle contraction and therefore preserving skill performance $[5,6]$. Previous studies have shown that sport drinks containing 6-8\% carbohydrate will facilitate absorption of solutes and water, and they will provide a source of energy to preserve glycogen reservoirs [7]. Protein has also been shown to promote fluid retention after rehydration [8-10], plasma volume expansion [11], and muscle protein synthesis [12].

Commercially available drinks on the market are made in an attempt to provide athletes with an optimized drink to rehydrate after exercise [13]. Commercial drinks, however, are expensive options and they are often not affordable for all athletes particularly those in low income countries. In addition to the costs, athletes tend to consume beverages rich in anabolic-energetic compounds and the latter has raised health concerns [14]. Therefore, there has been research on using available natural drinks as rehydrating options, such as tea, coffee, fruit juice and beer. There are, however, mixed results about the rehydrating potential of these drinks. Tea has been shown not to be more effective than plain water in rehydration and restoring fluid balance after exercise [15-18]. There is insufficient evidence supporting a superior rehydrating potential of coffee compared with other drinks [19]. Moreover, the current evidence does not suggest superior rehydration potential of juice in comparison with plain water [20,21]. Consuming beer and other alcoholic beverages after exercise led to an increased serum osmolality [22], most likely due to the diuretic effect of alcohol [22]. In addition, alcohol consumption leads to a long term negative effect on sport performance [23]. Therefore, there is yet no consensus on a suitable non-commercial drink for rehydration after strenuous exercise.

In this study, we examined yogurt drink as an inexpensive and widely available solution to rehydrate athletes after strenuous exercise. Yogurt is the product of milk fermentation with Lactobacillus bulgaricus and Streptococcus thermophiles [24]. Yogurt drink is made by mixing yogurt and chilled or iced water and has been variously described as "diluted yogurt", therefore it is a dairy product. The nutrient factors of this drink are similar to yogurt. Yogurt drink contains nutrients needed for post-exercise rehydration, such as electrolytes, carbohydrates and proteins. In addi- tion, yogurt drink has been a promising treatment for rehydrating children with diarrhea [25] and it therefore could be beneficial in cases of exercise-induced dehydration. In the current study we aimed to investigate the potential effect of yogurt drink in rehydrating and replenishing electrolyte losses in athletes after exercise in a hot and humid atmosphere.

\section{MATERIAL AND METHODS}

\section{Subjects}

In this cross-over trial, 10 athletes were randomly recruited from 100 healthy male football players. The inclusion criteria were age between 20 and 30 years, no previous intolerance of yogurt drinks and no history of metabolic or chronic diseases. Participants were asked to avoid exercise 24-hrs before the trial and to keep their routine diet and physical activity patterns. Every participant was evaluated in three separate days, while drinking water, a commercial drink or yogurt drink with a one-week wash out period between the interventions in order to compare their rehydrating potential. Samples of blood and urine were taken on four different time points on each day. Participants completed a consent form and the study was approved by the Ethics Committee of Tehran University of Medical Sciences with the following code: NO 1395/76925. The study has complied with the ethical principles for medical research involving human subjects outlined in the Declaration of Helsinki by the World Medical Association.

\section{Procedure}

Baseline: Subjects started the test by resting for 15 minutes and filling out questionnaires 1,2 and 3. After questionnaire completion, urine and blood samples $(1 \mathrm{ml})$ was collected from each participant for biochemical examinations. Body weight of all participants were measured subsequently without cloths (see flowchart of study in Fig. 1). In the next step, participants underwent the exercise workout.

Exercise: Participants performed a cycling exercise (70-80\% VO2max) at $35^{\circ} \mathrm{C}$ temperature and $70 \%$ humidity in the afternoon. Each exercise set was performed during a 10-minute period and subjects subsequently completed two or more sets until they experienced $2 \%$ reduction in body weight due to perspiration. After $2 \%$ weight reduction, their weight was subtracted from their baseline weight, and the amount of volume deficit was calculated.

Post-exercise: Participants took a 15-minute break and then urine and blood $(1 \mathrm{ml})$ samples were collected for biochemical examinations. In order to rehydrate, subjects were given a rehydration drink - yogurt drink 
or water (as the control) or a commercial sports drink in volume equivalent to their lost weight divided in 4 equal portions, which were taken 10 minutes apart. Thirty minutes later, blood $(1 \mathrm{ml})$ and urine samples were collected for biochemical examinations. Subjects rested for 3 hours and a final blood draw $(1 \mathrm{ml})$ and urine collection were collected [26-28].

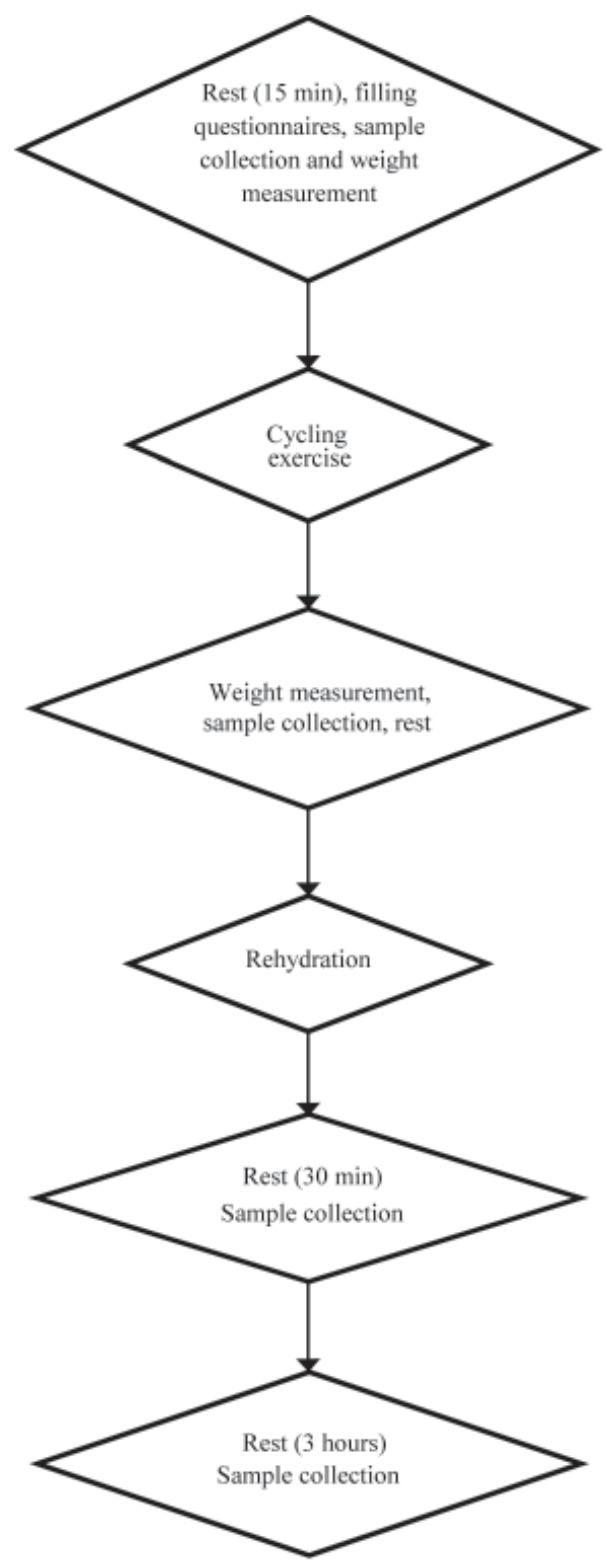

Fig. 1. Flowchat of the study steps

\section{Measures}

Measured parameters included anthropometric measurements, blood/serum and urine measurements. Body weight was measured by a standard scale (SOEHNLE, Germany). Whole blood was tested for hematocrit (\%) and blood sugar (mg/dl). In order to examine serum parameters, the whole blood was left undisturbed at room temperature for 30 minutes to clot. The clot was removed by centrifuging at $1500 \times \mathrm{g}$ for 10 minutes. The supernatant (serum) was collected for further analysis of serum osmolality (mos$\mathrm{mol} / \mathrm{kg}$ ) and sodium and potassium concentration $(\mathrm{mmol} / \mathrm{l})$. Urine test measurements included urine osmolality (mosmol/kg) and sodium, potassium and chloride concentration $(\mathrm{mmol} / \mathrm{l})$, as well as urine volume (ml).

\section{Yogurt drink}

The yogurt drink used in our study was a non-carbonated plain yogurt drink with no added ingredients (mint or pepper) manufactured by a high standard manufacturing Iranian company. The nutrient content of one cup $(240 \mathrm{ml})$ of the yogurt drink was as follows: protein: $4.25 \mathrm{gr}$, fat: $2.5 \mathrm{gr}$, carbohydrate: 6.25 gr, sodium: $625 \mathrm{mg}$, chloride: $875 \mathrm{mg}$.

\section{Commercial sports drink}

A standard commercial sports drink was used in the present study. One scoop (31 gr) was dissolved in one cup of water $(240 \mathrm{ml})$. The specific content of this drink was as follows: protein: $0 \mathrm{gr}$, fat: $0 \mathrm{gr}$, carbohydrate: $21 \mathrm{gr}$, sodium: $285 \mathrm{mg}$, chloride: $0 \mathrm{mg}$.

\section{Questionnaires}

Three separate questionnaires were given to the participants; questionnaire 1 consisted of eight questions including basic information such as age, medical history and experiencing any side effects after sports. Questionnaire 2 was a 24 hour dietary recall that evaluated the athletes' energy and nutrient intake in the past 24 hours which was asked by an expert in nutrition. Questionnaire 3 consisted of questions evaluating the taste and any side effects of drinking yogurt on the participants.

\section{Statistical analysis}

One-Way Analysis of Variances (ANOVA) was applied to each time point to investigate a significant difference among the three experimental groups. In case of a statistically significant difference, it was further analyzed by Bonferroni's post hoc test. SPSS 21.0 (IBM Corp., Armonk, NY, USA) was used for data analysis. The statistical significance was considered as $P<0.05$. The 24 -hour dietary recall was analyzed by the nutritionist IV software.

\section{RESULTS}

In this cross-over trial all the ten participants accomplished the three exercise regiments on three different days. The mean age (SD) and BMI (SD) of the athletes were 21.6 years $(0.8)$ and $23.16 \mathrm{~kg} / \mathrm{m} 2$ (2.84), respectively. There was no significant difference between the amount of calories and beverage intake between the participants in the past 24 hours prior to the experiment. 
Blood/serum parameters (Fig. 2), such as hematocrit $(\%)$, osmolality ( $\mathrm{mOsm} / \mathrm{Kg})$, serum glucose $(\mathrm{mg} /$ $\mathrm{dl})$, sodium and potassium concentrations ( $\mathrm{mmol} / \mathrm{L}$ ) were within a normal range for all participants at the beginning of the study and there was no significant difference among the three experimental groups. The group's mean values for blood/serum parameters remained within a normal range for all experimental groups at the end of the study (i.e., 3 hours after rehydration). There were no significant differences in hematocrit, serum osmolality and serum electrolyte levels between the three groups after rehydration with water, commercial drink or yogurt. However, the blood sugar decreased after rehydration with water which showed a significant difference $(P<0.01) 30$ minutes after rehydration compared to the commercial drink. However, rehydration with any of these three types of drinks could replenish post-exercise losses since the aforementioned blood/serum markers remained within a normal range.

Urine electrolytes (Fig. 3), including urine sodium, potassium and chloride concentrations ( $\mathrm{mmol} / \mathrm{L})$, osmo- lality $(\mathrm{mOsm} / \mathrm{Kg})$ and also urine volume $(\mathrm{ml})$ did not differ among groups at the beginning of the study. Yogurt drink compared to water significantly increased urine sodium (mmol/L) (138.8 \pm 93.4 vs. $90.0 \pm 50.7$, $\mathrm{P}<0.01)$, urine potassium $(\mathrm{mmol} / \mathrm{L})(105.6 \pm 55.1$ vs. $35.8 \pm 22.0, P<0.05)$ and urine chloride $(\mathrm{mmol} / \mathrm{l})$ $(113.2 \pm 28.4$ vs. $35.8 \pm 25.1 \mathrm{P}<0.01)$ early after rehydration (third sample collection). Rehydration with yogurt drink continued to show significant increases in urine sodium (mmol/L) $(162.5 \pm 58.1$ vs. $118.8 \pm$ 81.3, $\mathrm{P}<0.01)$, urine potassium ( $\mathrm{mmol} / \mathrm{L})(62.4 \pm$ 48.2 vs. $22.0 \pm 22.97, \mathrm{P}<0.05)$ and urine chloride $(\mathrm{mmol} / \mathrm{L})(88.6 \pm 45.0$ vs. $17.7 \pm 10.7, \mathrm{P}<0.001)$ concentrations compared to water as late as 3 hours after rehydration (fourth sample collection). Analysis of urine electrolytes showed that while rehydration with water significantly decreased the levels of urine sodium $(P<0.001)$, chloride $(P<0.01)$ and osmolality $(P<0.01)$, rehydration with yogurt drink was able to keep urine osmolality close to the baseline level. There was no significant difference in urine parameters between yogurt drink and the commercial drink.
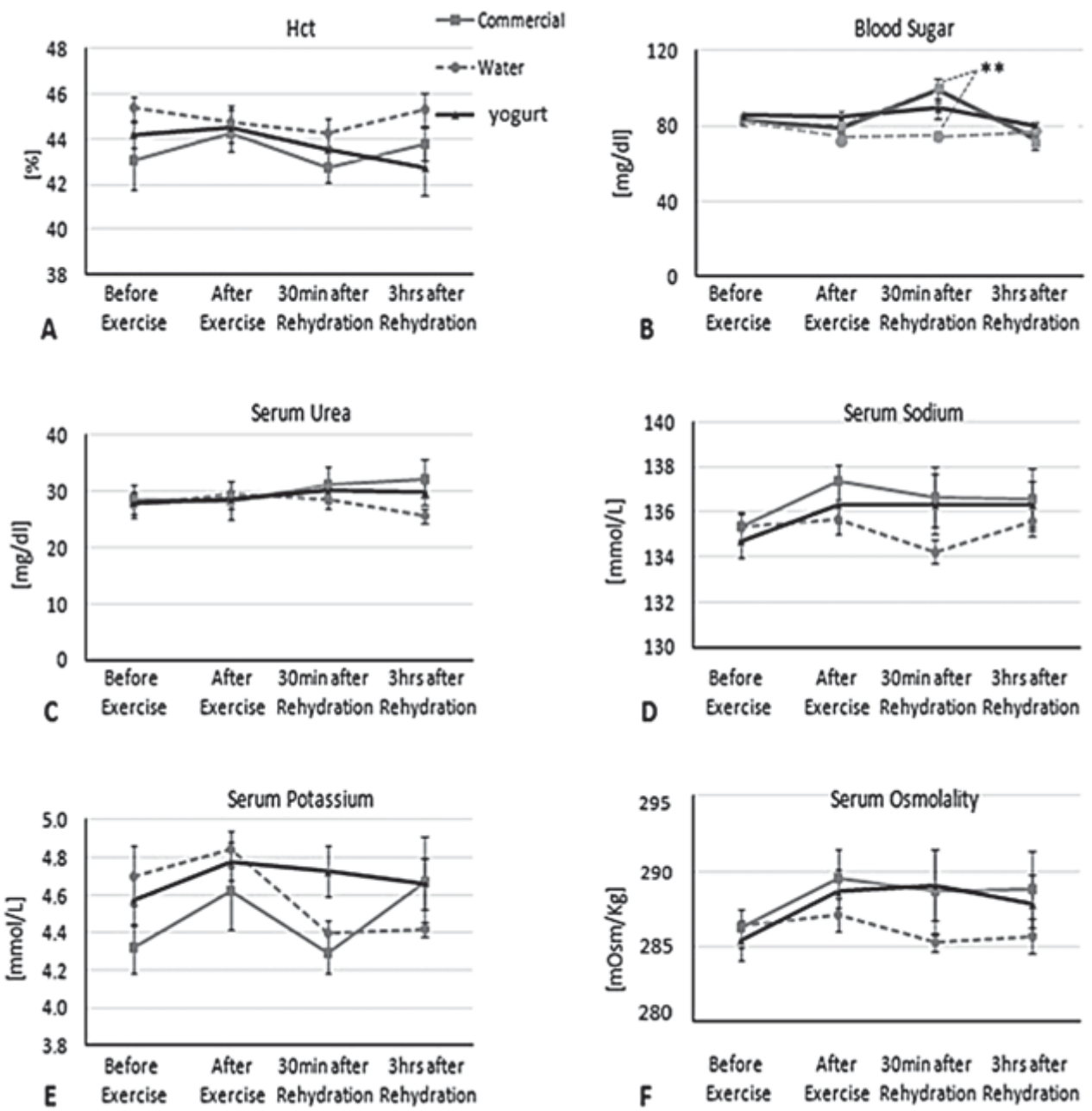

Blood hematocrit (A), blood sugar (B), serum urea (C), serum sodium (D), serum potassium (E) and serum osmolality (F) measured before exercise, immediately after exercise, shortly (30 minutes) after rehydration and long (3 hours) after rehydration. ${ }^{* *}$ in dicates $\mathrm{P}<0.01$.

Fig. 2. Blood parameters indicating the state of hydration during the trial 

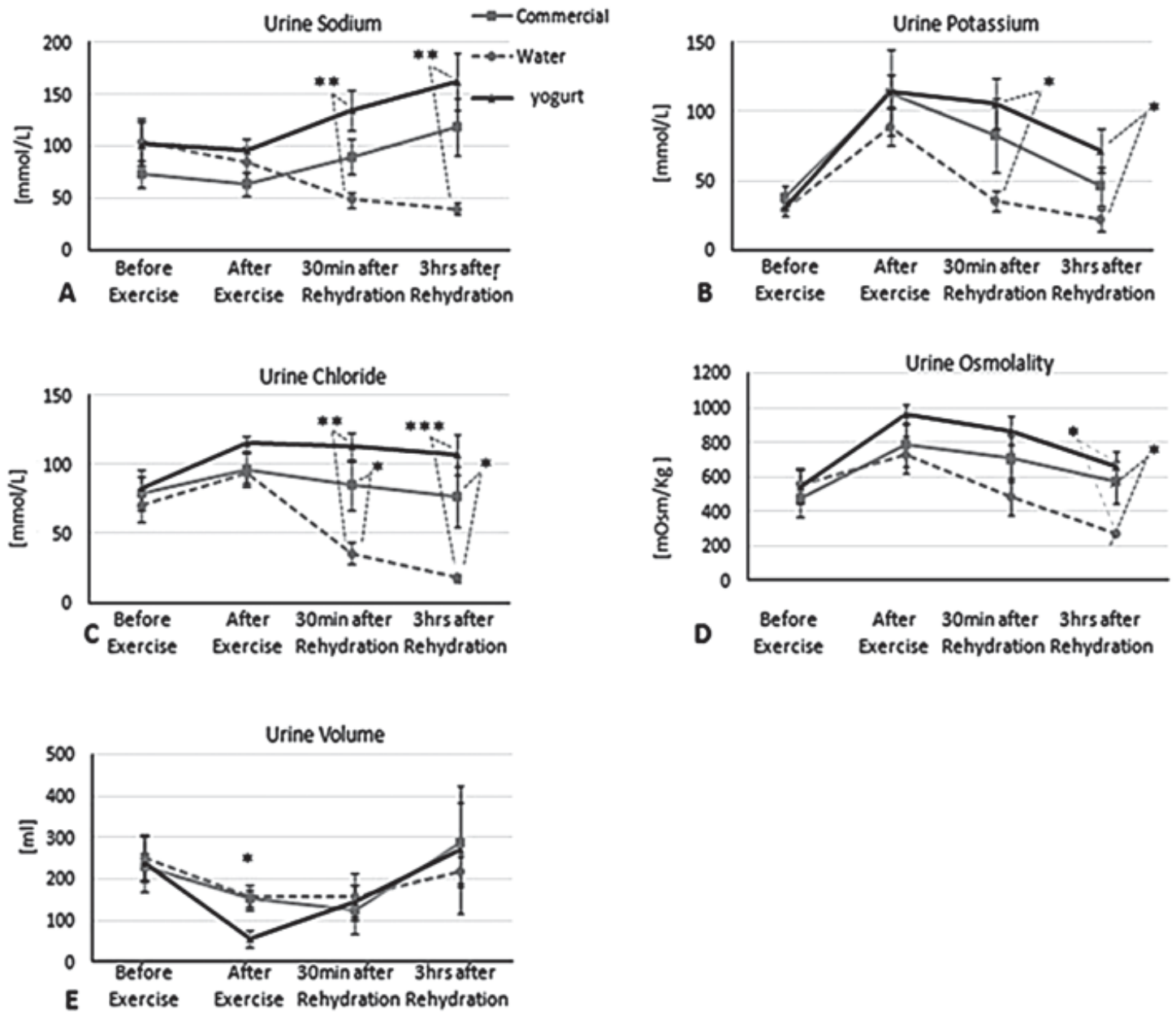

Urine sodium (A), urine potassium (B), urine chloride (C), urine osmolality (D) and urine volume (E) before exercise, immediately after exercise, shortly (30 minutes) after rehydration and long (3 hours) after rehydration. * indicates $\mathrm{P}<0.05$, ${ }^{* *}$ shows $\mathrm{P}<0.01$ and ${ }^{* * *}-\mathrm{P}<0.001$

Fig. 3. Urine parameters indicating the state of hydration during the trial

\section{DISCUSSION}

Our findings support the efficacy of yoghurt drink in replenishing the electrolyte loss of athletes at least during short term recovery after a workout session. The rehydration potential of yogurt drink is the same as that of commercial rehydration drinks. Since yogurt drink is a low-cost beverage, this will ensure a proper rehydration source for low-income countries to improve recovery after strenuous exercises for athletes in hot and humid environments. To our knowledge, this is the first study to examine the rehydration potential of yogurt drink after exercise, compared to water and a commercially available sports drink, and to analyze their effects on serum and urine electrolytes. Few studies have investigated the potential beneficial effects of yogurt drink on recovery after sports. Since oxidative stress arises immediately with the onset of anaerobic exercises [29], yogurt drink as a modulatory combination has been shown to decrease C-reactive protein and LDL compared to non-alcoholic beer and a carbohydrate drink after the Running based Anaerobic Sprint Test (R.A.S.T) in Taekwondo players [30].

In our study we have shown that yogurt drink is able to rehydrate athletes after $>2 \%$ weight loss caused by a strenuous exercise session in a hot and humid environment. Yogurt drink was compared to a commercial drink and water was considered as control. Focusing on serum parameters all three fluid rehydration choices were able to maintain levels of hematocrit, osmolality and serum sodium and potassium concentrations after exercise compared to baseline. In figure 2(B) blood sugar shows a more stable trend after rehydration with yogurt drink compared to the other two drinks. A raise in blood sugar after rehydration with the commercial drink could be related to the higher concentration of carbohydrate com- 
pared to yogurt drink. Furthermore, the effectiveness of yogurt drink is easily seen when one focuses on urine parameters; rehydration by yogurt drink leads to improvement in concentrations of multiple urine parameters, such as urine osmolality, urine sodium, potassium and chloride concentrations compared to water rehydration. The impact of yogurt drink in restoring urine electrolytes was much the same as the commercial drink and to some extent urine electrolytes such as sodium and chloride were higher after rehydration with yogurt drink compared to the commercial drink but the difference was not significant. This rehydration effect could be due to the appropriate combination of yogurt drink as mentioned before. Low urine electrolytes reflect physiological response of kidneys to low serum volume and high serum osmolality states and therefore they could be surrogate markers for dehydration [30]. In accordance to our findings, one study compared the effect of milk and a commercial drink on serum, urine and fluid balance, which showed that milk has a positive effect on fluid and electrolyte balance after exercise [31].

Rehydration could reflect homeostasis physiology where it aims to sustain serum parameter levels in expense of undulations in levels of urine electrolytes, and one could argue a more strenuous exercise, longer than 10 minute periods, may be required to unravel changes in serum electrolyte levels and osmolality. Effective rehydration by yogurt drink reflects the fact that yogurt drink provides important elements lost during exercise. Water and sodium are the two most important components that are lost in perspiration [32]. In addition, presence of proteins in rehydration fluids contributes to improved protein synthesis rates [33]. Yogurt has been shown to include concentration of $71.43 \mathrm{mg} / 100 \mathrm{gr}$ sodium and $5.02 \mathrm{gr} / 100 \mathrm{gr}$ proteins [34] whereas yogurt drink includes higher amounts of salt (sodium chloride) which would contribute to its electrolyte restoring potentials. Interestingly, other milk-derived drinks have been studied as post-exercise rehydration choices. Chocolate milk has been shown to be a good option for exercise recovery as it attenuates indices of muscle damage [35]. In addition, it has been shown that the effect of skimmed milk to promote fluid balance after exercise-induced dehydration has been more pronounced than a carbohydrate-electrolyte sports drink and therefore it has been discussed as a potential optimal rehydration drink [9]. This further highlights the role of milk- and yogurt-derived drinks for post-exercise rehydration.

There has been recently similar interest to study natural drinks as rehydration sources after strenuous exercises. For example, deep ocean mineral water has been shown to rehydrate and keep muscle performance in stationary biking in a warm and humid environment [27]. Furthermore, oral rehydration solution (ORS), full fat milk and semi-skimmed milk have been shown to be effective rehydrating options, but not cola, diet cola, hot tea, iced tea, coffee, lager, orange juice and sparkling water [28]. Honey drink has also been shown to improve muscle performance and glucose metabolism [26].

To our best knowledge this is the first study that examined the effect of yogurt drink on serum and urine electrolyte concentrations after strenuous exercise. Since the athletes underwent all the three trials, therefore the participants were considered as their own control, which increased the reliability of the results. However the study had certain limitations. Firstly, one limitation of the study is the short period of strenuous exercise. A longer regimen of strenuous exercise could better evaluate the rehydration potential of yogurt drink in more difficult exercise programs. Secondly, the present study only examined male athletes, and therefore the results could not be generalized to both sexes. Moreover, we did not include perspiration parameters in the current study which could help confirm the rehydrating potentials of yogurt drink. Further long-term studies could be designed to examine the effect of yogurt drink on the body composition, muscle glycogen storage and performance of athletes.

\section{CONCLUSION}

Our results indicate that yogurt drink, as a natural and affordable rehydration option, can be considered to restore fluid and electrolyte losses, caused by strenuous exercises in hot and humid environments. Furthermore, rehydration potential of yogurt drink is comparable to commercially available drinks

\begin{abstract}
Acknowledgments
This study is related to the project NO 1395/76925 from Student Research Committee, Shahid Beheshti University of Medical Sciences, Tehran, Iran. We also appreciate the "Student Research Committee" and "Research and Technology Chancellor" in Shahid Bheshti University of Medical Sciences for their financial support of this study.
\end{abstract}

The authors are grateful to the athletes for participating in the present study.

\section{Conflict of interests}

The authors declare no conflict of interest. 


\section{REFERENCES}

1. Cheuvront SN, Kenefick RW, Montain et al. Mechanisms of aerobic performance impairment with heat stress and dehydration. J App Physiol. 2010;109(6):1989-95.

2. Cheuvront SN, Kenefick RW. Dehydration: physiology, assessment, and performance effects. Compr Physiol. 2014;4(1):257-85.

3. Zoorob R, Parrish M-EE, O'Hara H, Kalliny M. Sports nutrition needs: before, during, and after exercise. Primary Care: 2013;40(2):475-86.

4. Bergeron MF. Hydration and thermal strain during tennis in the heat. Br J Sports Med. 2014;48 Suppl 1:112-7.

5. Jeukendrup AE. Carbohydrate feeding during exercise. Eur J Sport Sci. 2008;8(2):77-86.

6. Mitchell JB. Ingestion of carbohydrate during recovery in exercising people. Curr Opin Clin Nutr Metab Care. 2013;16(4):461-5.

7. Russell M, Kingsley M. The efficacy of acute nutritional interventions on soccer skill performance. Sports Med. 2014;44(7):957-70.

8. James LJ, Clayton D, Evans GH. Effect of milk protein addition to a carbohydrate-electrolyte rehydration solution ingested after exercise in the heat. Br J Nutr. 2011;105(3): 393-9.

9. James LJ, Gingell R, Evans GH. Whey protein addition to a carbohydrate-electrolyte rehydration solution ingested after exercise in the heat. Journal of Athletic Training. 2012;47(1):61-6.

10. James L. Milk protein and the restoration of fluid balance after exercise. Med Sport Sci. 2012;59:120-6.

11. Goto M, Okazaki K, Kamijo et al. Protein and carbohydrate supplementation during 5-day aerobic training enhanced plasma volume expansion and thermoregulatory adaptation in young men. J App Physiol. 2010;109(4):1247-55.

12. Phillips SM, Van Loon LJ. Dietary protein for athletes: from requirements to optimum adaptation. J Sports Sci. 2011;29 Suppl 1:S29-38.

13. Baker LB, Jeukendrup AE. Optimal composition of fluidreplacement beverages. Comprehensive Physiology. 2014;4(2):575-620.

14. Kargarfard M, Shariat A, Lam E et al. Prevalence and Perceptions Toward Anabolicandrogenic Steroid Use Amongst University Students. AMB 2015,42(2),61.

15. Chang CQ, Chen YB, Chen ZM, Zhang LT. Effects of a carbohydrate-electrolyte beverage on blood viscosity after dehydration in healthy adults. Chin Med J. 2010;123(22):3220-5.

16. Utter AC, Quindry JC, Emerenziani GP, Valiente JS. Effects of rooibos tea, bottled water, and a carbohydrate beverage on blood and urinary measures of hydration after acute dehydration. Res Sports Med. 2010;18(2):85-96.

17. Wong SH, Chen Y. Effect of a carbohydrate-electrolyte beverage, lemon tea, or water on rehydration during shortterm recovery from exercise. Int J Sport Nutr Exerc Metab. 2011;21(4):300-10.

18. Wong SH, Sun FH, Huang WY, Chen YJ. Effects of beverages with variable nutrients on rehydration and cognitive function. Int J Sports Med. 2014;35(14):1208-15.

19. Ishikawa $\mathrm{T}$, Tamura $\mathrm{H}$, Ishiguro $\mathrm{H}$ et al. Effect of oral rehydration solution on fatigue during outdoor work in a hot en- vironment: a randomized crossover study. J Occup Health. 2010;52(4):209-15

20. Miller KC, Mack G, Knight KL. Electrolyte and plasma changes after ingestion of pickle juice, water, and a common carbohydrate-electrolyte solution. J Athl Train. 2009;44(5):454-61.

21. Kalpana K, Lal PR, Kusuma DL, Khanna GL. The effects of ingestion of sugarcane juice and commercial sports drinks on cycling performance of athletes in comparison to plain water. Asian J Sports Med. 2013;4(3):181-9.

22. Hobson RM, Maughan RJ. Hydration status and the diuretic action of a small dose of alcohol. Alcohol Alcohol. 2010;45(4):366-73.

23. Barnes MJ. Alcohol: impact on sports performance and recovery in male athletes. Sports Medicine. 2014;44(7):909-19.

24. Adolfsson O, Meydani SN, Russell RM. Yogurt and gut function. Am J Clin Nutr. 2004;80(2):245-56.

25. Eren M, Dinleyici EC, Vandenplas Y. Clinical efficacy comparison of Saccharomyces boulardii and yogurt fluid in acute nonbloody diarrhea in children: a randomized, controlled, open label study. Am J Trop Med Hyg. 2010;82(3):488-91.

26. Ahmad NS, Ooi FK, Saat Ismail M, Mohamed M. Effects of Post-Exercise Honey Drink Ingestion on Blood Glucose and Subsequent Running Performance in the Heat. Asian $\mathrm{J}$ Sports Med. 2015;6(3):e24044.

27. Keen DA, Constantopoulos E, Konhilas JP. The impact of post-exercise hydration with deep-ocean mineral water on rehydration and exercise performance. J Int Soc Sports Nutr. 2016;13:17.

28. Maughan RJ, Watson P, Cordery PA, Walsh NP, Oliver SJ, Dolci A, et al. A randomized trial to assess the potential of different beverages to affect hydration status: development of a beverage hydration index. Am J Clin Nutr. 2016;103(3):717-23.

29. Afzalpour M, Bashafaat $H$, Shariat $A$ et al. Plasma protein carbonyl responses to anaerobic exercise in female cyclists. Int $\mathrm{J}$ App Exer Physiol. 2016;5(1):53-8.

30. Shiranian A, Darvishi L, Askari G et al. The effect of different beverage consumption (dough, non-alcoholic beer, carbohydrated replacement drink) on performance, lipids profile, inflammatory biomarkers after running-based anaerobic sprint test in taekwondo players. Intl J Prev Med. 2013;4(Suppl 1):S5-S10.

31. Watson P, Love TD, Maughan RJ, Shirreffs SM. A comparison of the effects of milk and a carbohydrate-electrolyte drink on the restoration of fluid balance and exercise capacity in a hot, humid environment. Eur J Appl Physiol. 2008;104(4):633-42.

32. Shirreffs SM, Maughan RJ. Volume repletion after exerciseinduced volume depletion in humans: replacement of water and sodium losses. Am J Physiolo. 1998;274(5):F868-F75.

33. Beelen M, Burke LM, Gibala MJ, van Loon LJ. Nutritional strategies to promote postexercise recovery. Int J Sport Nutr Exerc Metabol. 2010;20(6):515-32.

34. Kailasapathy K, Chin J. Survival and therapeutic potential of probiotic organisms with reference to Lactobacillus acidophilus and Bifidobacterium spp. Immunol Cell Biol. 2000;78(1):80-8.

35. Pritchett K, Pritchett R. Chocolate milk: a post-exercise recovery beverage for endurance sports. Med Sport Sci. 2012;59:127-34. 